\title{
Aide apportée par la télédétection à la cartographie des prairies permanentes
}

\author{
Colette M. GIRARD \\ I.N.A. Paris-Grignon - Laboratoire de Botanique et Ecologie végétale, F 78850 Thiverval-Grignon
}

RÉSUMÉ

\begin{abstract}
Les prairies permancntes représentent 40 p. 100 de la surface agricole utile en France; or, cette surface importante est actuellement encore mal connue, tant dans sa distribution géographique que dans la composition floristique de sa vćgétation et ses qualités agronomiques.

L'étude présentée ici correspond à la mise au point d'unc méthode de diagnostic et de cartographic des prairies et pâturages appliquée à la gestion et à l'aménagement. Cette méthode comporte l'utilisation conjointe de données botaniques (relevés phytosociologiques) et de données de télédétection (analyses spectrales au sol ct données satcllitaires).

Les données de terrain servent à définir des unités tant sur le plan phytosociologique que radiométrique. Elles guident le seuillage des données satellitaires. Un exemple de cartographic est donné et discuté.
\end{abstract}

Mots clés additionnels : Composition colorée, étude diachronique, données satellitaires, phytosociologie, pixel, radiomètres, traitement des données.

Grassland represents about $40 \%$ of the agricultural area in France. This large area is still poorly known as much for its spatial distribution as for its botanical composition and agronomical value.

This paper describes the claboration of a method for cvaluating and mapping grassland as an aid to management. This method combined botanical (field samples) and remote sensing data (spectral measurements in the ficld and satellite data).

Data collected on the ground were used for botanical and spectral description of units and also for choosing threshold values for satellite data handling. An example of mapping is given and discussed.

Additional key words: Color composite, data handling, multitemporal studies, phytosociology, pixel, radiometers, satellite data.

\section{INTRODUCTION}

La télédétection a en France environ 12 ans d'âge. C'cst déjà beaucoup pour un ensemble de techniques sans cesse en amélioration. A peine des techniques viennent-elles d'être élaborées et testées qu'elles sont déjà remplacées par d'autres plus sophistiquées.

L'agronomie a fait l'objet d'études d'application de la télédétection surtout sur les pcuplements cultivés (HOLBEN et al., 1980 ; TUCKER et al., 1980). La botanique, quant à elle, n'en a quasiment pas suscitées. Les prairies permanentes pâturćcs, dont l'étude de la végétation ressort du domaine de la botanique et dont les applications concernent l'agronomic, nous ont semblé un sujet d'ćtude particulièrement intéressant, où la télédétection peut rendre des services importants. En effet, cette dernière permet d'obtenir une information analytique très précise sur les proprićtés spectrales des pcuplements végétaux ct, en même temps, une information synthétique spatialisée sur les communautés végétales et leur distribution (STOVE \& HuLME, 1980).

C'est l'emploi conjoint des méthodes botaniques d'analyse et de définition des groupements végétaux et des méthodes de télédétection qui a été tenté, de façon à définir une méthode de diagnostic et de cartographic des prairies et pâturages.

Les méthodes d'analyse et de diagnostic s'appuyant sur des données phytosociologiques ayant été présentées dans d'autres publications (DELPECH, 1978), c'est surtout l'aspect' cartographique qui sera développé.

\section{RÉGION ÉTUDIÉE. MÉTHODES D'ÉTUDE}

La zone d'étude correspond à une surface de $200 \mathrm{~km}^{2}$ située en Auxois et centréc sur la ville de Vitteaux (Côted'Or), en pleine zone herbagère, où les prairies permanentes représentent 54 p. 100 de la surface totale (SCEES, 1980). 
Ces prairies sont réparties principalement dans le fond ct sur les flancs cle vallées parallèles, de direction NNW-SSE, séparées par des plateaux étroits où sont localisées les cultures. L'esquisse géologique montre l'intercalation de niveaux calcaires plus ou moins durs avec des marnes liasiques. Cette alternance de roches de duretés différentes donne un relief caractéristique (fig. 1).

Dans cette zone, 95 stations de prairie ont été étudiées en 1980, 1981, 1982. Les techniques utilisées sont les suivantes :

1) Lc relcvé botanique, portant sur une surface de 16 à $50 \mathrm{~m}^{2}$, où sont notées les espèces végétales affectées d'un coefficient d'abondance-dominance (BRAUN-BLANQUET, 1926). Le stade de végétation des plantes cst aussi indiqué pour les mois d'avril, mai, juin et juillet auxquels chaque station a été successivement observéc.

D'autre part, des renseignements concernant les paramètres environnementaux (exposition, pente, humidité de surface du sol, $\mathrm{pH}$, piétinement...) sont indiqués sur la fiche de description phytoćcologique (fig. 2).

2) Les mesures de réflectance par radiométric à $1,50 \mathrm{~m}$, verticalement, au-dessus de la surface du sol.

Deux radiomètres différents ont été utilisés : l'un (ISCO du laboratoire de Chimie biologique de l'INA P.G.) a un angle d'ouverture large $\left(90^{\circ}\right)$ et des bandes spectrales nombreuses, de 380 à $1500 \mathrm{~nm}$, de 25 à $50 \mathrm{~nm}$ de large.

L'autre (BIOMETRE de la NASA) a un angle d'ouverture étroit $\left(12^{\circ}\right)$ et seulcment 2 bandes spectrales larges : 630 $690 \mathrm{~nm}$ et $760-900 \mathrm{~nm}$.

Ces instruments, qui mesurent l'énergic réfléchie ou émise, permettent de collecter une information spectrale sur les surfaces visćcs (KIMES et al., 1980 ; TUCKER et al., 1980 ; GiRARD, 1982).
3) Les données du satellite LANDSAT :

Parmi les différentes images disponibles sur la zonc-test, celle du 29 avril 1976 de la scène 213027 a été retenue, car avril correspond à la reprise de l'activité chlorophyllienne de la végétation. Les contrastes sont alors plus accentués entre les zones où la reprise d'activitć chlorophyllienne est précoce et celles où clle est tardive.

D'autre part, pour les prairies où la reprise de l'activité chlorophyllienne est commencée, on observe une forte différenciation en fonction des phénophases colorées de certaines espèces. Par exemple, les prairies humides à Cardamine pratensis $\mathrm{L}$. se distinguent des prairies sèches à Primula veris L. comme l'illustrent les courbes de réflectance acquises sur le terrain (fig. $3 a$ et $3 b$ ).

En l'absence de données satellitaires d'avril 1980, celles d'avril 1976 ont été retenues. 1976 étant une année à déficit hydrique marqué, les différences de conditions de drainage entre les stations y sont plus fortes qu'cn 1980.

Les données captées par le satellite LANDSAT le sont dans les bandes spectrales suivantes:

- canal 4 : 500-600 nm, bande vert du visible.

- canal 5 : 600-700 nm, bande jaune-orange du visible.

- canal 6: 700-800 nm, bande du début du proche infrarouge.

- canal 7: 800-1 $100 \mathrm{~nm}$, bande du proche infra-rouge.

Les dimensions du point élémentaire (pixel) «vu » à un instant donné sont de $56 \times 79 \mathrm{~m}$. Ce qui pose le problème du passage des données de terrain au pixel, qui est en cours d'étude.

Pour chaque pixel, dans chaque bande spectrale, sont cnrcgistrées des valcurs de flux réfléchi (luminance énergétique spectrale). Bien qu'il s'agisse de données numériques,

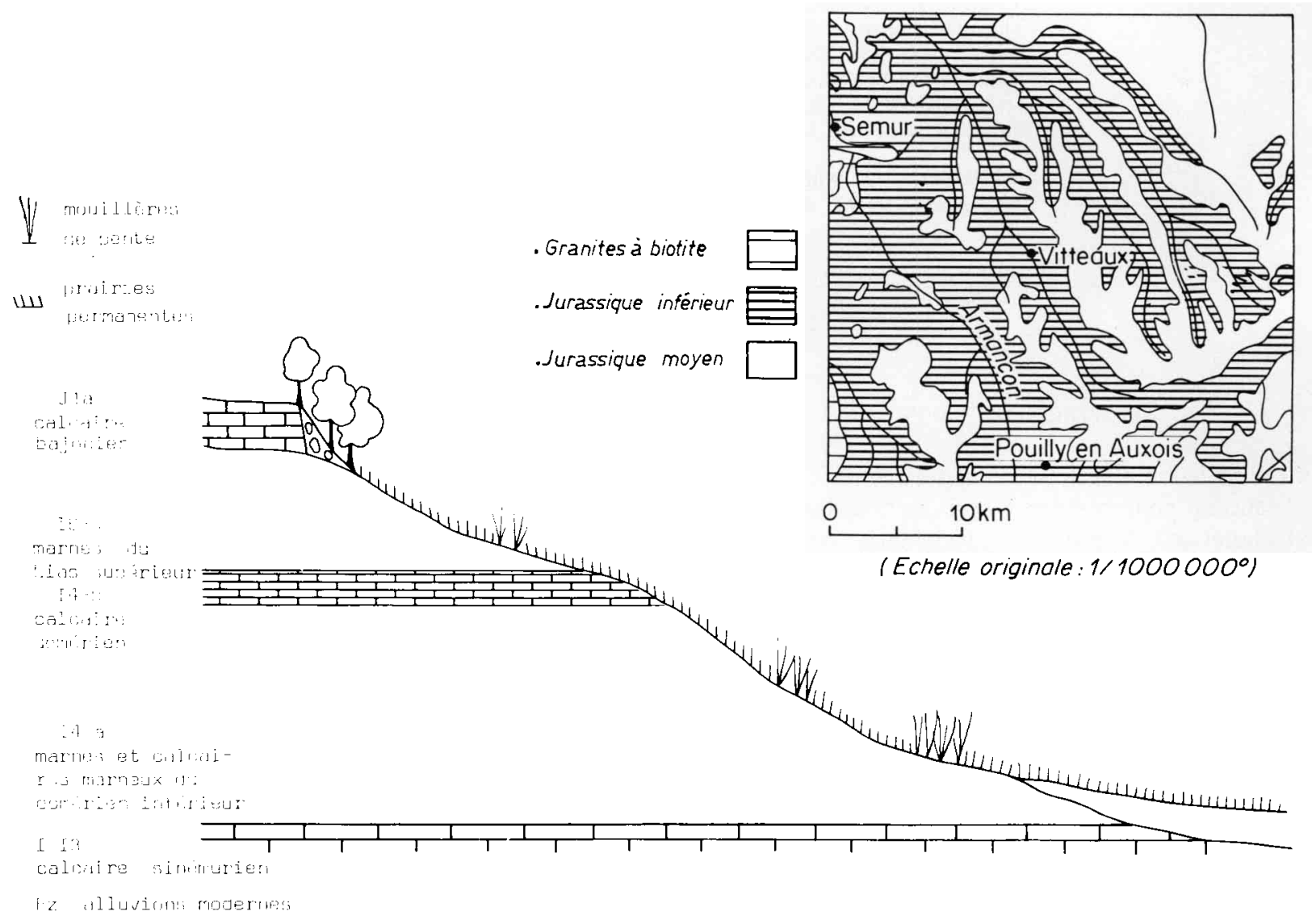

Figure 1 


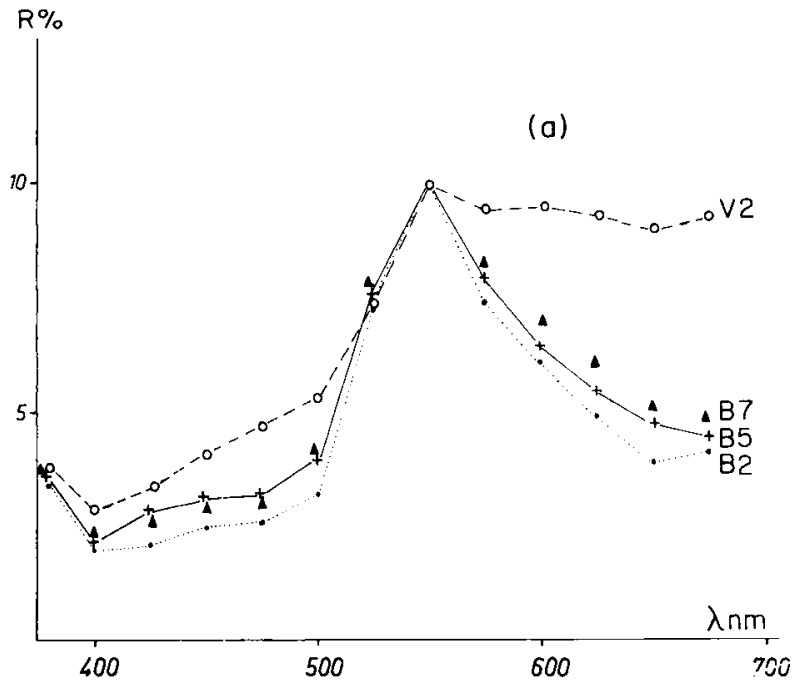

Figure 3

a) Courbes de réflectance pour différentes prairies en avril 1980 (courbes calées dans le visible sur $\lambda=550 \mathrm{~nm}, R=10 \%$ ).

b) Courbes de réflectance pour différentes prairies en avril 1980 (courbes non calées).

a) Reflectance curves for different grasslands in April 1980 (Adjusted curves in the visible on $\lambda=550 \mathrm{~nm}, R=10 \%$.) b) Reflectance curves for different grasslands in April 1980. (Non adjusted curves.)

$B_{2}: \cdots \cdots \cdots . \quad \quad$ : Prairie fraîche $\grave{a}$ Lolium perenne $L$ Moist grassland with Lolium perenne $L$.

ces données ne doivent pas être considérées comme des données quantitatives. En effet, il n'y a pas, lors de l'enregistrement, de source de référence permettant de les caler les unes par rapport aux autres. La valeur 100 pour l'enregistrement d'avril 1976 ne correspond pas à la valeur 100 d'un enregistrement fait à une autre date.

\section{INTERPRÉTATION DES DONNÉES DE TERRAIN}

\section{A. Les données radiométriques}

L'analyse des données spectrales obtenues avec les 2 radiomètres montre que la réflectance traduit bien l'intensité de l'activité photosynthétique ainsi que la densité de recouvrement du sol par les plantes (fig. $3 a$ et $3 b$ ). La prairie $V_{2}$, où l'activité chlorophyllienne des végétaux est faible ainsi que le recouvrement du sol par les végétaux, a des valeurs de réflectance plus fortes dans le visible et plus faibles dans le proche infra-rouge que les autres prairies.

L'étude du cornportement spectral des prairies permanentes a fait l'objet d'une autre publication et ne sera pas discutéc ici (GIRARD, 1982).

\section{B. Les données phytosociologiques}

Les données phytosociologiques n'ont pas encore été toutes traitées par l'analyse factorielle des correspondances (LACOSTE \& Roux, 1971). Un début de classement, complété par la comparaison avec d'autres travaux phytosociologiques (ROYER et al., 1975 ; LONCHAMP, 1977), met en évidence, en première approximation, la relation entre les stations étudićes et les alliances suivantes :
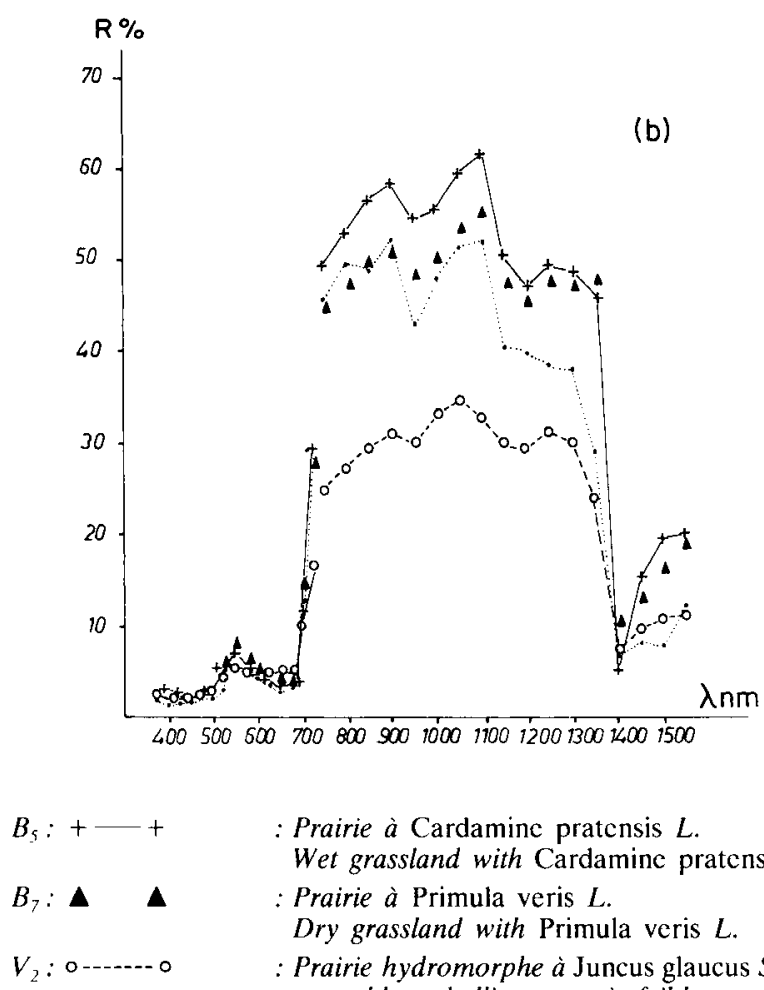
: Prairie à Cardamine pratensis $L$. Wet grassland with Cardamine pratensis $L$. : Prairie à Primula veris $L$. Dry grassland with Primula veris $L$.
: Prairie hydromorphe à Juncus glaucus Sibth. peu chlorophyllienne et à faible recouvre- ment du sol par la végétation.
Damp grassland with Juncus glaucus Sibth. at low chlorophyll content and soil cover.

1. Mesobromion erecti Br. Bl. ct Moor 48

2. Cynosurion Tx. 47

3. Arrhenatherion elatioris W. Koch 26

4. Agropyro Rumicion crispi North 40

5. Calthion Tx. 37.

L'interprétation des données phytosociologiques est en cours et sera développéc dans un autre travail.

\section{Les données agronomiques}

L'interprétation des facteurs écologiques ainsi que des modalités d'utilisation correspondant aux différentes stations permet de les regrouper selon 5 classes équivalentes aux précédentes :

1. Prairies pâturécs héliophiles et calcicoles sur sols peu profonds à drainage excessif.

2. Prairies fraîches pâturées sur sols profonds généralement bien drainćs.

3. Prairies fraîches fauchées sur sols profonds généralement bien drainćs.

4. Prairies humides généralement pâturćes sur sols profonds surtout cutrophes à drainage insuffisant.

5. Prairies pâturécs sur sols profonds assez longtemps engorgés.

Une étude de la valeur agronomique potenticlle des prairics a aussi été tentée à partir des relevés stationnels des 3 annćcs. La mćthode employćc est la suivante (DELPECH, 1978, 1979)

On calcule, pour chaque station, la somme $\mathrm{S}$ de tous les coefficients d'abondance-dominance des différentes espèces présentes dans le relevé (le cocfficient + est considéré comme égal à 0,1 ).

La somme $F$ de tous les coefficients d'abondance-dominance des espèces fourragères est ensuite calculée. A 
l'intérieur de celle-ci, on calcule Fp, somme des coefficients d'abondance-dominance des espèces fourragères productives. Enfin, on calcule $\mathrm{T}$ somme des coefficients d'abondance-dominance des espèces toxiques.

Les rapports correspondants: $\mathrm{F} / \mathrm{S}, \mathrm{Fp} / \mathrm{S}$ ct $\mathrm{T} / \mathrm{S}$ sont ensuite calculés.

Ces valeurs permettent d'apprécier la valeur potentielle d'une prairie. Plus F/S et Fp/S sont élevés tandis que T/S cst bas, meillcure est la prairic.
Mais cette appréciation est théorique, car on ne tient compte que de l'abondance-dominance des espèces. Cela ne permet pas d'apprécier des phénomènes, tels que le surpâturage, qui affectent l'ćtat physiologique et le stade do développement des végétaux et dont les coefficients d'abondance-dominance ne tiennent pas compte.

Ayant établi les réserves nécessaires concernant les rapports et leur signification, il est intéressant de les comparer entre eux (tabl. 1).

\section{TABLEAU 1}

Rapports $\mathrm{Fp} / \mathrm{S}$ et $\mathrm{F} / \mathrm{S}$ pour toutes les stations de prairies permanentes. $F p / S$ and $F / S$ ratios for all grassland stations.

Graminées

Arrhenatherum elatius (L.) J. \& C. Presl. Dactylis glomerata L.

Festuca pratensis Huds.

Lolium perenne $\mathrm{L}$.

Phleum pratense $\mathrm{L}$.

Poa pratensis L.

Poa trivialis $\mathrm{L}$.

Trisetum flavescens (L.) P. B.

Listc des espèces classćcs en Fp

\section{Légumineuses}

Medicago sativa $\mathrm{L}$.

Onobrychis viciifolia Scop.

Trifolium pratense $\mathrm{L}$.

Trifolium repens $\mathrm{L}$.
Autres familles

Crepis taraxacifolia Thuill.

Daucus carota L.

Pimpinella major (L.) Huds.

Plantago lanceolata $\mathrm{L}$.

Sanguisorba minor Scop.

Taraxacum gr. officinale Weber.

Tragopogon pratensis $\mathbf{L}$.

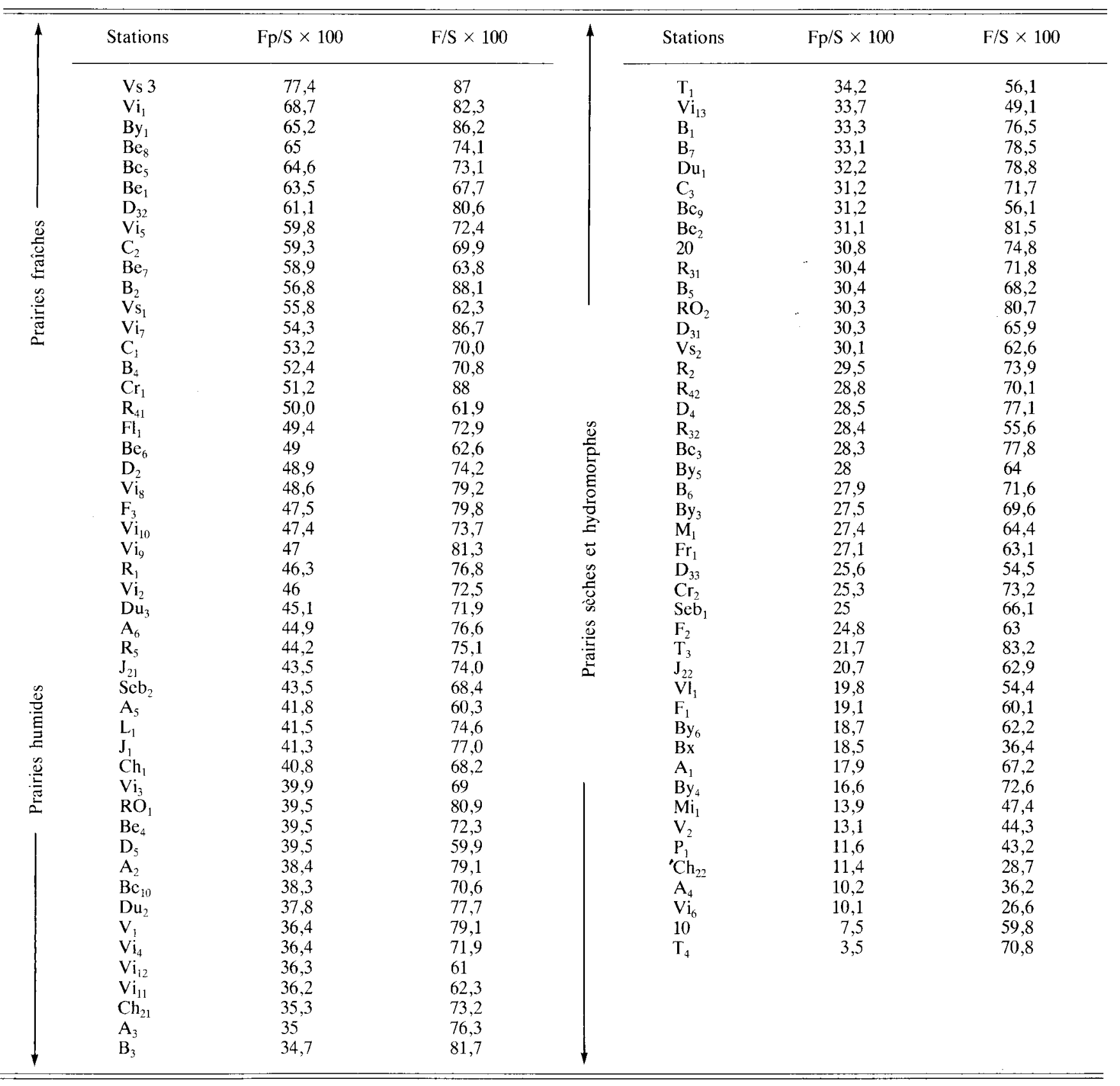


Ce tableau montre le classement des stations en fonction de $\mathrm{Fp} / \mathrm{S}$. Nous avons préféré cc rapport à $\mathrm{F} / \mathrm{S}$ qui scmblait moins représentatif des prairies. Il apparaît sur ce tableau que les prairies fraîches pâturées et fauchées ont des valeurs de $\mathrm{Fp} / \mathrm{S}$ élevées, tandis que les prairies pâturées très sèches ou hydromorphes ont les valcurs les plus faibles.

Les espèces fourragères ayant la plus forte productivité croissent en abondance dans les stations où la profondeur du sol est suffisante et le drainage est correct sans être excessif. La distribution spatiale des prairies selon leur valeur agronomique est fonction de la distribution spatiale des paramètres écologiques que sont la profondeur du sol et son drainage.

Les techniques de télédétection vont être utilisées pour réaliser une cartographic des prairies permanentes selon des critères agro-écologiques.

\section{LES DONNÉES RADIOMÉTRIQUES SATELLITAIRES-CARTOGRAPHIE}

Différentes classifications ont été entreprises à partir des donnécs LANDsAT du 29 avril 1976, sur le système de traitement de clonnées TRIAS de l'Institut Géographique National (DENEGRE et al., 1980) ; 3 programmes ont été successivement employés.

\section{A. « Mesures ponctuelles de radiance »}

Sur une composition colorćc, obtenue par la superposition des canaux 5, 6 et 7 , affectés chacun d'une couleur différente (fig. 4), on a repéré les pixels correspondant à des stations étudiées sur le terrain ct on a noté les valeurs de luminance dans chacun des canaux.

Ces valeurs ne sont pas comparables quantitativement à celles obtenues sur le terrain dans des bandes équivalentes, principalement parce que les mesures sont faites à des distances au sol très différentes $(900 \mathrm{~km}$ pour LANDSAT, $1,50 \mathrm{~m}$ pour les radiomètres) et que l'on ne tient pas compte des modifications spectrales dues à l'atmosphère. C'est ce qu'illustre la figure 5 qui donne la position de différentes stations de terrain en fonction du rapport:

$$
\frac{\text { valeur du canal } 7 \text { - valeur du canal } 5}{\text { valeur du canal } 7+\text { valeur du canal } 5}
$$

respectivement pour les luminances de LANDSAT et le flux énergétique réfléchi pour l'Isco. Ce rapport permet de bien différencier les végétaux à fonction chlorophyllienne active.

Les mesures de radiométric IsCo ont été faites sur le terrain entre le 24 et le 30 avril 1980 et correspondent à des stades phénologiques comparables pour les prairies, mais à des conditions de luminance non identiques à celles mesurées par LANDS.AT.

Bien qu'il s'agisse de données numériques, ces données ne doivent pas être considérées comme des données quantitatives absolues.

\section{B. «Zones-tests »}

Ce programme fait intervenir la connaissance que l'on a du terrain. Elle consiste en la délimitation, sur l'écran de visualisation, des surfaces correspondant à des pixels où sont situées les stations étudiées sur le terrain.

Ce traitement automatique n'est possible que si ces

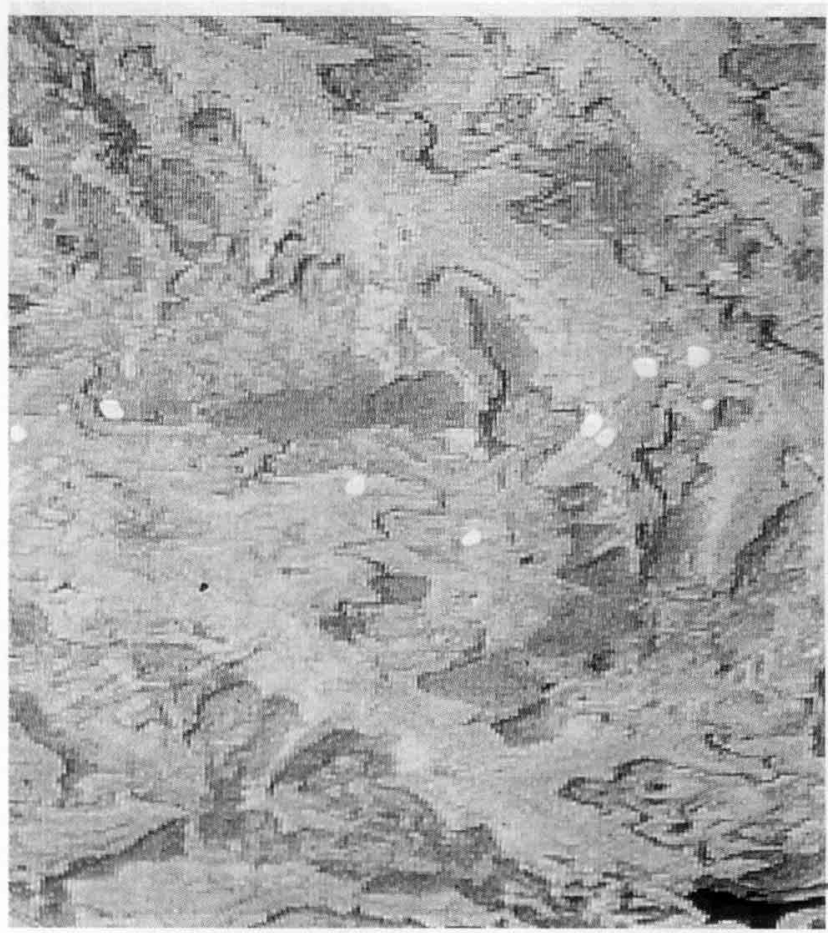

Figure 4

Composition colorée d'une partie de la. scène 213-27 du 29/4/1976.

Bleu : Sols nus. Villes.

Vert : Forêts.

Jaune : Prairies permanentes.

Noir : Etangs.

Les taches blanches correspondent à des zones-tests sur le terrain. Color composite of a part of scene 213-27 on 4/29/1976.

Blue :Bare soils. Towns.

Green : Forests.

Yellow :Grasslands.

Black : Lake.

White spots represent test areas in the fields.

surfaces ont des valeurs de luminance homogènes dans les différents canaux LANDSAT. Une vingtaine de stations homogènes sur une grande surface a été retenue. Ces stations se regroupent dans les 5 alliances phytosociologiques indiquées au chapitre III,B.

Ensuite, chaque pixel du fragment de scène étudić est exploré et rattaché par un calcul de distance (propre à l'I.G.N.) à l'une ou l'autre «zone-test » (fig. 6).

Cette méthode appliquéc à la scène d'avril 1976 permet, comme on va le voir, de distinguer les prairies sèches, les prairies fraîches et les prairies plus ou moins hydromorphes. La méthode a l'avantage d'être très rapide puisque effectuéc par ordinateur.

Ce type de classification ne donne que des résultats globaux et ne peut traduire la complexité de ce qui cst observé sur le terrain, à un niveau de perception différent. Elle montre néanmoins une distinction possible entre plusieurs ensembles de prairies.

\section{C. « Seuillage »}

Ce dernier programme, contrairement aux précédents qui utilisent l'information contenue dans les 4 canaux LAND. SAT, est basé sur l'étude d'un seul canal, ici le canal 7.

Les 5 grandes unités agro-écologiques ont des valeurs de luminance différentes sur le canal 7. On a déterminé des classes sur l'histogramme des luminances. Elles ont été 


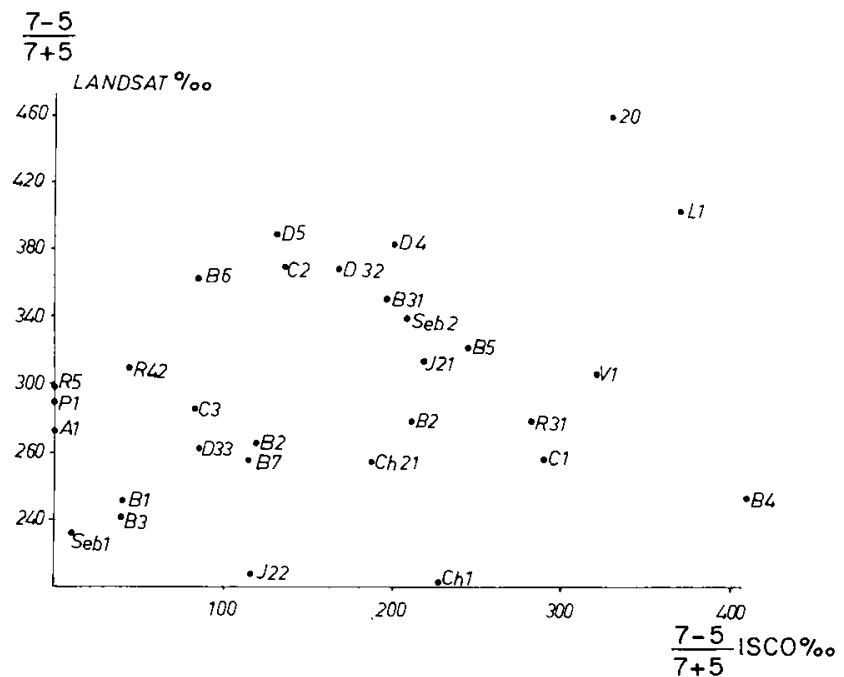

Figure 5

Comparaison des luminances LANDSAT avec les flux énergétiques réfléchis mesurés par le spectroradiomètre de terrain Isco pour différentes stations $(7=800-1100 \mathrm{~nm} ; 5=600-700 \mathrm{~nm})$.

Comparison of LANDSAT luminances with reflected fluxes measured with ISCO spectroradiometer, "in situ", for different stations.

ajustées en fonction de la vingtaine de stations retenues précédemment.

La difficulté d'un tel programme réside dans le choix des valeurs de luminance délimitant les classes. Le « seuillage» peut être tout à fait arbitraire, il n'est vraiment satisfaisant qu'éclairé par la connaissance du terrain qui permet un choix judicicux des seuils.

Le tableau 2 donne les unités reconnues par cette classification, avec les couleurs correspondantes. Ce seuillage, jugé satisfaisant pour l'interprétation, a été visualisé et fixé sur un support stable, coloré (CROMALIN) (fig. 7).

Le seuillage réalisé sur le canal 7 donne de bons résultats pour les raisons suivantes :

1) Dans les longueurs d'onde correspondant à ce canal, les végétaux chlorophylliens bien turgescents réfléchissent beaucoup le rayonnement solaire. En avril, seules les prairies permanentes sont chlorophylliennes et décelables par le satellite. Elles auront les valeurs de luminance les plus

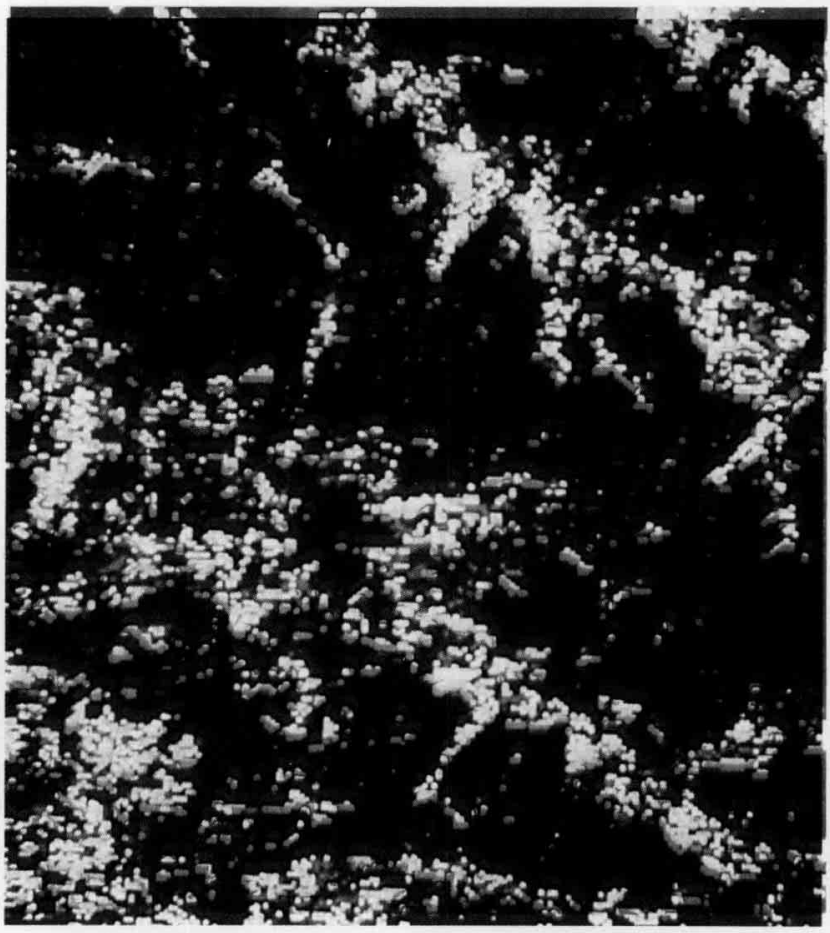

Figure 6

Résultat du traitement par zones-tests.

Noir : Plateaux sans prairies.

Rouge : : Prairies sèches.

Jaune : Prairies fraiches.

Blanc $\} \quad$ : Prairies fraîches et mésohygrophiles.

Results of classification by test-areas.

Black : Plateaux without grasslands.

Red : Dry grasslands.

Yellow : Moist grasslands.

$\left.\begin{array}{l}\text { White } \\ \text { Blue }\end{array}\right\} \quad$ : Moist and wet grasslands.

fortes et pourront être plus facilement ćtudiées vis-à-vis des autres couverts végétaux.

2) Le canal 7 a une dynamique plus grande que les autres canaux. Les variations de luminance dans les canaux 4,5 et 6 apparaîtront moins nettement que sur le canal 7. C'est pourquoi les variations de réflectance dans le visible, liées

TABLEAU 2

Découpage en classes à partir des valeurs de luminance du canal 7 de la scène 212027 du 29 avril 1976 et signification de ces classes. Class thresholds from channel 7 luminance values on scene 212027 taken on April 29th 1976, and significance of classes.

\begin{tabular}{|c|c|c|}
\hline $\begin{array}{l}\text { Valcurs de flux } \\
\text { réfléchi }\left({ }^{*}\right)\end{array}$ & $\begin{array}{l}\text { Codage coulcur sur } \\
\text { la visualisation }\end{array}$ & Type d'occupation du sol, ou type de \\
\hline $60-75$ & Vert & Sols cultivés et forêts \\
\hline $76-82$ & Rouge & Pacages à moutons, pelouses sèches \\
\hline $83-89$ & Rouge & Prairies pâturées sèches \\
\hline $96-98$ & Saumon & Prairies sur sol très hydromorphe \\
\hline $99-103$ & Bleu & Prairies sur sol hydromorphe \\
\hline $104-106$ & Blanc & Prairies pâturées fraîches \\
\hline $107-109$ & ou & classe intermédiaire \\
\hline $110-113$ & Crème & Prairies fauchées, entretenues \\
\hline
\end{tabular}

(*) Ces valeurs ne correspondent pas à des valcurs de flux telles qu'elles sont mesurées sur le terrain et ne sont pas exprimées dans une unité car elles sont le résultat de traitements complexes.

Les critères de distinction des grands types prairiaux sont basés sur le régime hydrique du sol (présence ou absence d'hydromorphie, drainage) le mode d'exploitation (pâture, fauche). 
aux phénophases colorées des prairies permanentes, se traduiront par des variations de luminance qui risquent d'être perçues comme un bruit plus que comme un signal. Si le pixel était de dimensions plus petites, comme c'est le cas pour LANDSAT D et le futur satellite SPOT $(20 \times 20 \mathrm{~m})$, et si les bandes spectrales étaicnt mieux placées, les phénophases colorées seraient peut-être discernables.

Par contre, sur le canal 7, ces variations de luminance, liées à des différences d'activité chlorophyllienne, de turgescence et de recouvrement du sol par les végétaux chlorophylliens, apparaîtront plus nettement. Elles vont servir à distinguer les divers ensembles prairiaux.

3) Enfin, il n'est pas sûr que la distance utiliséc dans le programme précédent pour établir les classes soit forcément bien adaptée à l'êtude des phénomènes considérés. On peut lui préfércr un «seuillage » réalisé d'après les choix du thématicien, qui, en privilégiant certaines luminances, fera micux ressortir les unités de prairic définies à partir du travail de terrain.

Les résultats des classifications automatiques ne sont pas des cartes, mais des images en coulcur, des compositions colorées. Le thématicien doit les interpréter pour les transformer en cartes.

Sur le cromalin de 1976, des surfaces ont été délimitées en fonction des couleurs de l'image et interprétées en fonction de la connaissance du terrain. La figure 8 montre l'esquisse à l'échelle du $1 / 150000^{\circ}$ des ensembles prairiaux qui, pour des raisons de graphisme, ont été regroupés en :

- prairies sèches et fraîches, d'une part,

- prairies humides et hydromorphes, d'autre part.

Sur cette esquisse, des ensembles complexes ont été définis, ils regroupent des zones cultivées avec des prairies fraîches ou des prairies humides.

\section{INTERPRÉTATION DE DONNÉES LANDSAT DIACHRONIQUES}

A partir des données du canal 7 d'une nouvelle image de la scène (6 septembre 1977), un scuillage a ćté réalisć et la composition colorée résultante a été interprétéc visucllement comme celle d'avril 1976.

Ensuite, les 2 interprétations ont été comparées sur une petite surface agrandic au 1/25000 pour plus de lisibilité (fig. 9). On peut faire la correspondance pixel à pixel de 2 images de la même scène, mais on ne peut mettre en correspondance les valeurs de luminance des 2 images, l'élévation du soleil et les conditions atmosphériques n'étant pas les mêmes. La comparaison des 2 interprétations n'a pas été faite avec l'assistance de l'ordinateur pour plusicurs raisons :

1) Le stade phinologique des végétaux n'est pas du tout le même, ce qui retentit sur les valeurs de luminance.

2) Les prairies ne sont pas dans le même ćtat physiologique et n'ont pas les mêmes caractéristiques spectrales. En conséquence, certaines prairies ont été classées dans des unités différentes sclon les scuillages.

3) Compte tenu de la date (septembre), certaines cultures sont confondues avec les prairies permanentes et la discrimination d'unités de prairies sur la base des seules valcurs de luminance se fait mal sur les données de septembre.

Scules, la réflexion et la connaissance du terrain permettent de trancher et de savoir à quel ensemble il faut finalement rattacher les différents pixels.
Une rapide évaluation des surfaces correctement classées par une simple superposition des unités montre que 41,4 p. 100 seulement de la surface ont été correctement classés. Cela montre bien la limitation d'une comparaison automatique des données. L'ordinateur, s'il avait été employé, n'aurait pas pu classer correctement 58,6 p. 100 des données, puisqu'il ne peut procéder qu'à une simple superposition des 2 séries de données. Cela est dû non à la qualité du logicicl mais à l'impossibilité de classer tous les pixels correctement par les valeurs de luminance.

L'interprétation par le botaniste a surtout porté sur les zones mal classées. Le résultat est donné sur la figure $9 d$. Les contours des unités sont plus précis, en particulier dans lc cas des forêts, des prairics humides et hydromorphes pour lesquelles subsistait une assez grande différence de contour entre les 2 images LANDSAT. Le choix des ensembles prairiaux a ćté fait en fonction des données botaniques et ćcologiques de terrain.

D'autrc part, une zone de cultures a pu être identifićc grâce à l'interprétation des 2 classifications. Cette zone apparaît en classe mixte forêts - cultures en $1977 \mathrm{ct}$ en classe mixte prairies humides - cultures en 1976. L'interprétation conduit à en faire une unité homogène de cultures.

En ce qui concerne les prairies sèches de haut de pente, l'interprétation des 2 classifications permet de les localiser plus précisément. Elles apparaissent comme: forêts cultures une annéc et prairies fraîches l'autre. Compte tenu :

1) des variations climatiques observées entre les 2 annécs: sécheresse en 1976, humidité moyenne en 1977 ,

2) des variations dans l'ćtat physiologique des plantes des prairics entre avril et septembre,

3) de l'errcur apportée par les ombres portées soulignant la bordure ouest des reliefs, lcur contour a pu être établi en s'aidant de la connaissance du terrain.

Une évaluation grossière du niveau de la productivité des prairies a pu être réaliséc ensuite en se basant sur les résultats de l'interprétation de la répartition des prairies permanentes, d'une part, et sur la connaissance d'une relation entre certains paramètres écologiques et la classe de productivité, d'autre part ( $\$$ III C).

En effet, on a observé que les prairies contenant les espèces les plus productives sur le plan agronomique étaient situćes dans les meilleures conditions de profondeur de sol ct de drainage, c'est-à-dire dans les classes: prairies fraîches, prairies humides et dans la classe mixte: culturesprairics (tabl. 1).

Les prairies sèches et les prairies sur sols hydromorphes ont une moindre productivité fourragère ; quant aux forêts et cultures, elles n'ont, par définition, pas de productivité fourragère.

C'est à partir de cette constatation que l'on a tracé la figure 10. Elle est aussi au 1/25000 et résulte de la correspondance entre classes:

Forêts et culturcs: pas de productivité fourragère.

Prairies sèches et prairies hydromorphes : faible productivité fourragère.

Prairies fraîches et prairies humides : bonne productivité fourragc̀re.

\section{CONCLUSIONS}

L'exemple qui vient d'être donné pour une petite région de l'Auxois montre qu'il est possible d'utiliser des données satcllitaires de télćdétection dans la cartographie d'ensem- 


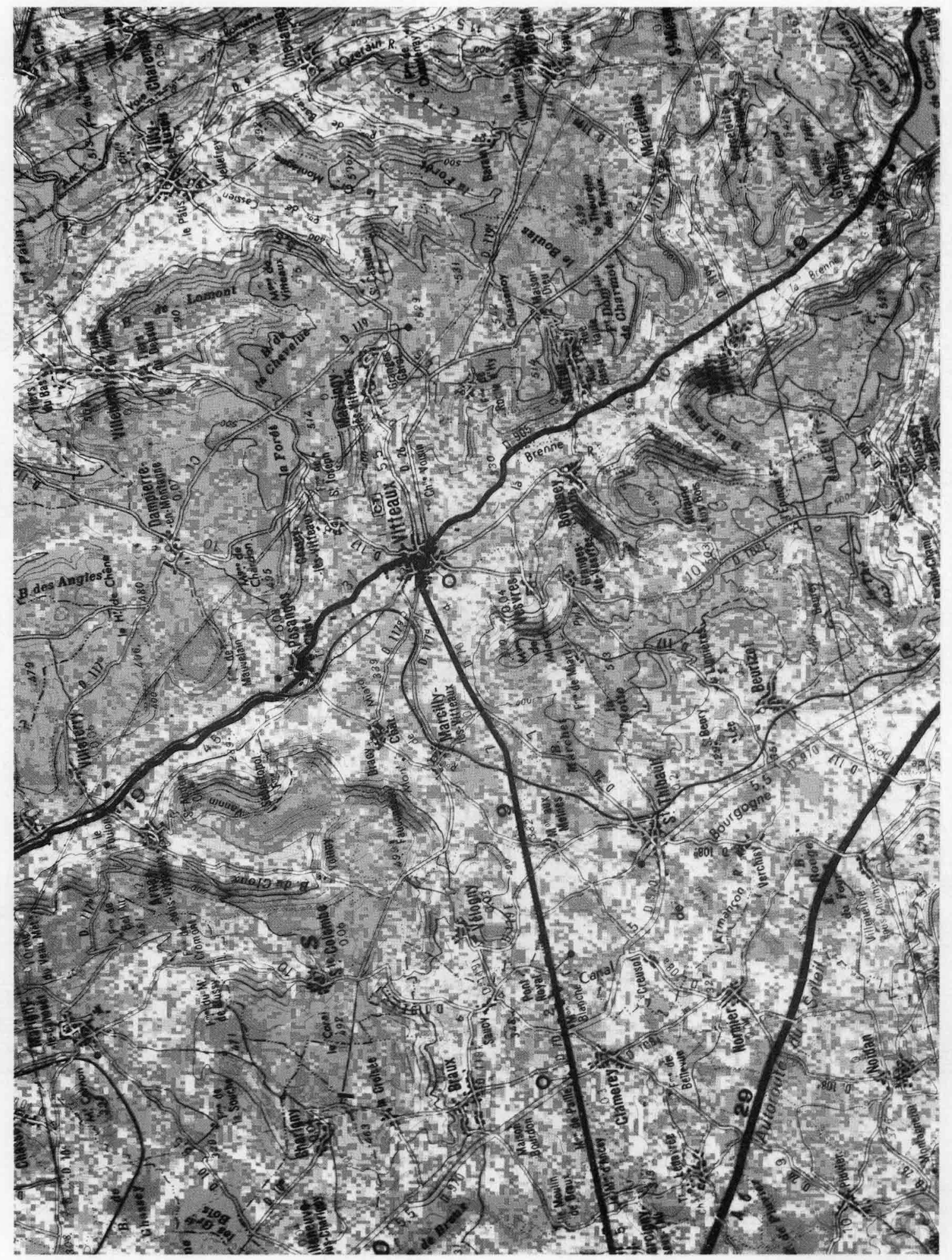

Figure 7

Résultat du «seuillage». (Pour la légende, cf. tabl. 2).
Result of threshold classification. A key to the colours is given in table 2. 


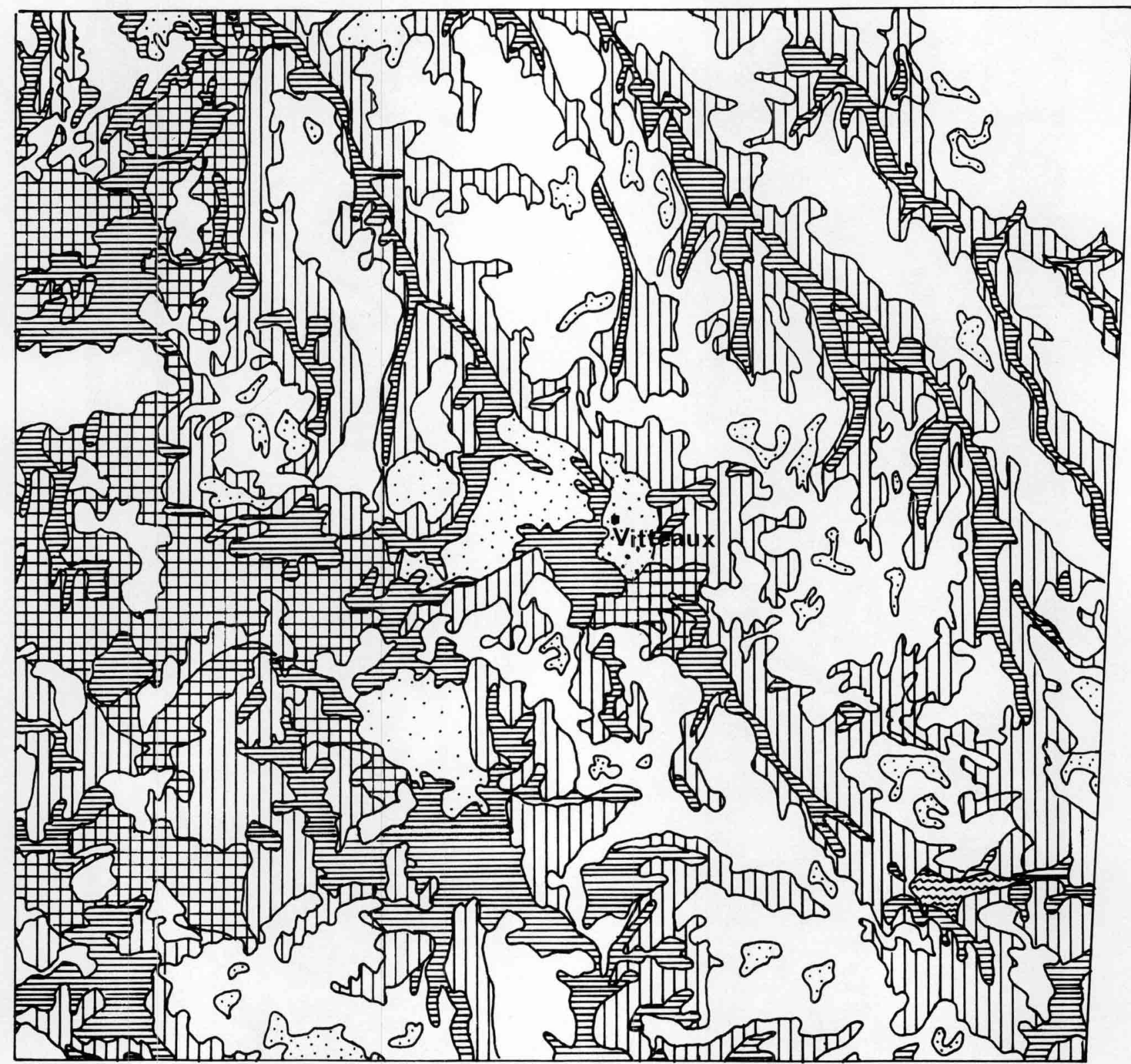

Figure 8

Esquisse au 1/150 000 des unités de prairies permanentes et d'occupation des sols à partir des données LANDSAT d'avril 1976.

Drawing at 1/150 000 of grassland units and types of land use from LANDSAT data of April 1976.

$\begin{array}{ll}\square & \text { Forêts, cultures } \\ & \text { Forests, crops } \\ \because \because \because{ }^{2} & \text { Cultures, prairies sèches } \\ \text { Crops, dry grasslands }\end{array}$

Prairies sèches et fraîches Dry and moist grasslands TाTा Prairies humides et cultures

11 Wet grasslands and crops
Prairies humides et sur sols hydromorphes 

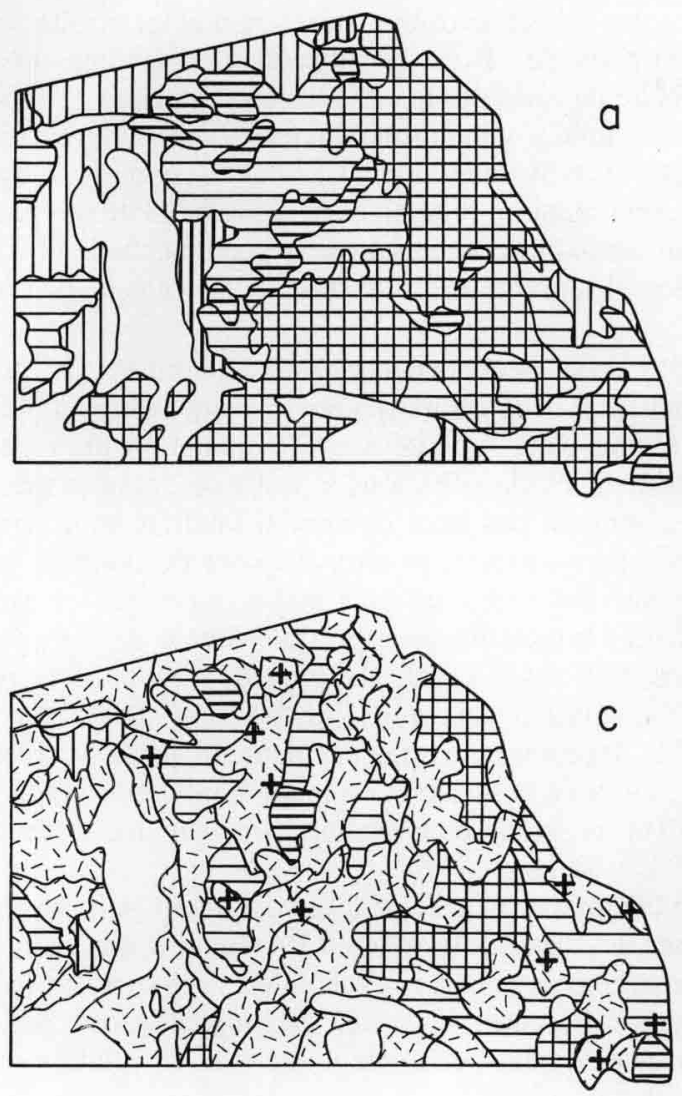

Figure 9

Comparaison des données Landsat d'avril 1976 et septembre 1977.

a) Interprétation des données de 1976.

b) Interprétation des données de 1977.

c) Superposition des données des deux dates. Seules ont été représentées les surfaces référencées identiquement en a) et b).

d) Interprétation des deux ensembles de données.

Comparison of April 1976 and September 1977 Landsat data.

a) Interpretation of 1976 data.

b) Interpretation of 1977 data.

c) Superposition of the two sets of data. Only areas identically referenced in a) and b) are represented.

d) Interperetation of the two sets of data.
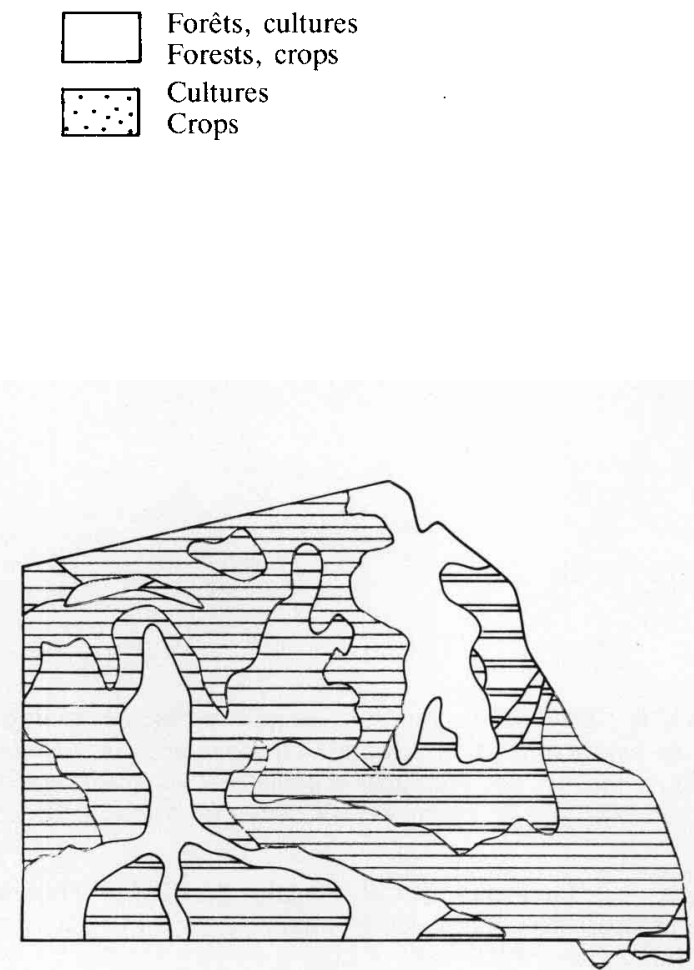
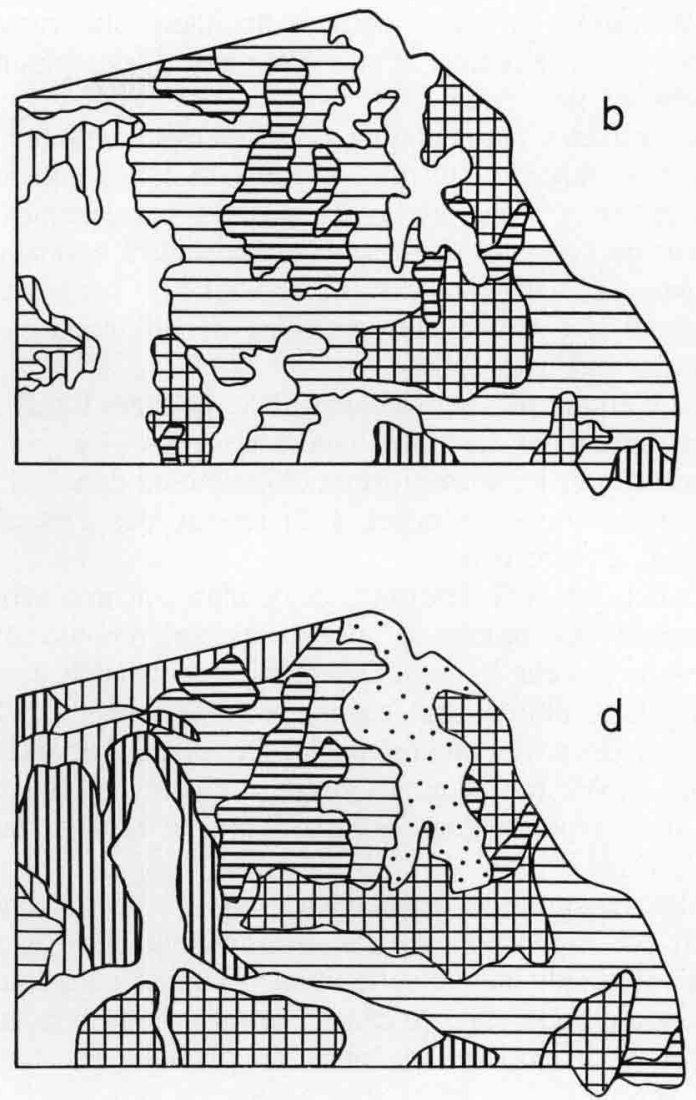

Figure 10

Carte du niveau de productivité fourragère des prairies élaborée à partir des ensembles prairiaux agro-écologiques.

Map of forage productivity level based on agroecological grassland units.

Forêts, cultures

Forests, crops

Prairies à basse productivité fourragère

Grasslands with a low forage productivity

Mosaïque de cultures et prairies

Mosaic of crops and grasslands

Prairies productives de bonne qualité

High forage productivity grasslands 
bles prairiaux, car elles permettent, dans unc certaine mesure, de spatialiser et d'extrapoler $\left({ }^{*}\right)$ des données stationnclles de terrain.

Les méthodes automatiques de classement, étayées par une connaissance du terrain, fournissent très rapidement une des bases d'une carte des prairies permanentes en fonction de certaines de leurs caractéristiques botaniques, édaphiques et agronomiques. Le résultat paraît satisfaisant au niveau des statistiques agricoles départementales ou cantonales par exemple.

Il est d'autant plus satisfaisant que les données traitées au départ présentent de forts contrastes entre les prairies permanentes et les autres formes d'occupation du sol, d'une part, et de forts contrastes à l'intérieur des ensembles prairiaux, d'autre part.

En effet, en avril, les forêts de feuillus ont une activité chlorophyllienne réduite ce qui entraîne une réponse relativement forte dans le canal 5 et relativement faible dans le canal 7. Les cultures sont encore peu développées et leur activité chlorophyllienne est faible. De même, l'abondance de sols cncore nus dans les zones cultivées entraîne unc assez forte réponse dans le canal 5 et une réponse faible dans le canal 7.

Seules les prairies permanentes ont un recouvrement végétal par rapport au sol nu suffisamment élevé et une activité chlorophyllienne suffisamment intense pour fournir une réponse faible dans le canal 5 et forte dans le canal 7.

En ce qui concerne septembre, les phénomènes sont en partie inversés. Les forêts sont encore en activité chlorophyllienne ainsi qu'une partic des cultures. Les prairies permanentes, en particulier celles situées sur les hauts de pente et dans les fonds de vallées (ces prairics sousexploitées présentent beaucoup de matière sèche qui masque les parties chlorophylliennes), ont une activité chlorophyllienne faible. A cette période, on risque de confondre les prairies sèches et les chaumes secs, ainsi que les prairies fraîches et les plantes sarclées encore en place. Lcs données satellitaires de septembre doivent être utilisées en complément des données d'avril et ne doivent pas être utilisées seules, car elles conduiraient à de trop grandes incertitudes de classification.

La répartition dans l'espace des ensembles prairiaux est, dans cette région, liée principalement à certaines caractéris-

(*) On distingue la spatialisation qui concerne une extension dans l'espace de pixels d'aspect semblable de l'extrapolation qui consiste à regrouper par interprétation des pixels dans des ensembles préalablement définis. tiques des sols et se trouve en relation assez étroite avec la géomorphologie. Il est possible de réaliser une première approche de classification des terroirs prairiaux, s'appuyant sur des unités géomorphologiques bien différenciées et facilement individualisables. Ces unités géomorphologiques paraissent aussi en relation avec la productivité des prairies, ce qui a conduit à une esquisse à grande échelle $(1 / 25000)$ d'ensembles prairiaux en fonction de lcur niveau de productivité.

S'il y avait indépendance cntre les unités de prairies permanentes et la géomorphologie, ce qui est possible dans une région plate soumise à une exploitation prairiale plus diversifiée, les classifications à partir de données satellitaires ne seraicnt pas aussi bonnes. Il faudrait pour arriver à des résultats corrects, pouvoir disposer de données satellitaires sur des pixcls de plus petite dimension et avec de meilleures caractéristiques spectrales que ceux des premiers LANDSAT. C'est le cas de satcllites de ressources terrestres de $2^{\mathrm{c}}$ génération tels que LANDSAT ou SPOT. Ce futur satellite français aura effectivement un pixel de $20 \mathrm{~m}$ sur $20 \mathrm{~m}$, en visée verticale et en mode multibande, et de $10 \mathrm{~m}$ sur $10 \mathrm{~m}$, en mode panchromatique, pour une trace au sol de $60 \mathrm{~km}$ de large.

On comprend qu'avec de telles données il soit possible de réaliser des classifications assistées tenant mieux compte de différences plus fines entre les unités prairiales, en particulier des différences liécs aux phénophascs colorées. Ces données devraient permettre un suivi de l'évolution saisonnière des prairics.

Il faut maintenant souhaiter que d'autres travaux se poursuivent dans la même voie, combinant des investigations de terrain très précises et des traitements de données satellitaires et aéroportées diachroniques, de façon à définir de nouveaux descripteurs et d'élaborer une méthode cartographique plus rapide et plus cfficace.

Reçu le 24 février 1983. Accepté le 17 octobre 1983.

\section{REMERCIEMENTS}

Ce travail a pu être réalisé grâce au support financier du Centre National d'Etudes Spatiales, dans le cadre d'une convention de recherches. Il a bénéficié des moyens de traitement de données de l'Institut Géographique National qui a gracieusement réalisé les différents traitements d'images dans le cadre d'un mémoire de fin d'étude de l'Ecole Nationale des Sciences Géographiques (ENSG).

\section{RÉFÉRENCES BIBLIOGRAPHIQUES}

Braun-Blanquet J., 1926. Une reconnaissance phytosociologique dans le Briançonnais. Bull. Soc. Bot. Fr., 73.

Cromalin. Process and equipment manual.

Delpech R., 1978. Essai d'appréciation de la valeur agronomique à partir des données phytosociologiques. In Bournerias M. et al., "Les groupements de prairies et leurs satellites dans la vallée inondable de l'Oise". Coll. phytosociol. V "La végétation des prairies inondables ». Lille 1976. J. Cramer, Vadug. 131-136.
Delpech R., 1979. Réflexions sur quelques problèmes biologiques soulevés par l'exploitation pastorale en montagne. In «Utilisation par les ruminants des pâturages d'altitude et parcours méditerranéens ». I.N.R.A., 10 $10^{c}$ Journées du Grenier de Theix, 1978, 45-56.

Denègre J., Lummaux J. C., Poulain J., 1980. Traitements d'images spatiales; méthodes et applications à l'I.G.N. France. Bull. Inf. I.G.N., 80/3 (42), 18-24. 
Girard C. M., 1982. Estimation of phenological stages and physiological states of grasslands from remote-sensing data. Vegetatio, 48 , 219-226.

Holben B. N., Tucker C. J., Fan C. J., 1980. Spectral assessment of soybean leaf area and leaf biomass. Photogramm Eng. Remote Sensing, 46 (5), 651-656.

Kimes D. S., Smith J. A., Ranson K. S., 1980. Vegetation reflectance measurements as a function of solar zenith angle. Photogramm. Eng. Remote Sensing, 46 (12), 1563-1573.

Lacoste A., Roux M., 1971. L'analyse multidimensionnelle en phytosociologie et écologie : application à des données de l'étage sub-alpin des Alpes-Maritimes. Oecol. Plant., 6 (4), 353-371.

Lonchamp J. P., 1977. Etude phytoécologique et essai de typologie des prairies permanentes de l'Auxois. Thèse Doct. Sci. Biol., Fac. Besançon, 123 p. + Annexes.
Royer J. M., Rameau J. C., Bidault M., 1975. Apport de la phytosociologie à la connaissance des territoires phytogéographiques. Applications à la Bourgogne calcaire. C.R. $99^{e}$ Congr. Natl. Soc. Savantes. Besançon, 1974.

S.C.E.E.S., 1980. Annuaire de statistiques agricoles 1980 (résultats 1979).

Stove G. C., Hulme P. D., 1980. Peat resource mapping in Lewis, using remote sensing techniques and automated cartography. Int. J. Remote Sensing, 1 (4), 319-344.

Tucker C. J., Elgin J. H., McMurtrey J. E., 1980. Relationship of crop radiance to alfalfa agronomic value. Int. J. Remote Sensing, 1 (1), 69-75. 